\title{
The influence of baryons on the chiral phase transition in QCD.
}

\author{
M.N. Chernodub and B.L. Ioffe \\ Institute of Theoretical and Experimental Physics, \\ B. Cheremushkinskaya 25, 117218 Moscow, Russia
}

\begin{abstract}
A qualitative analysis of the chiral phase transition in QCD with two massless quarks and non-zero baryon density is performed. It is assumed that at zero baryonic density, $\rho=0$, the temperature phase transition is of the second order. Due to a specific power dependence of baryon masses on the chiral condensate the phase transition becomes of the first order at the temperature $T=T_{\mathrm{ph}}(\rho)$ for $\rho>0$. At temperatures $T_{\text {cont }}(\rho)>T>T_{\mathrm{ph}}(\rho)$ there is a mixed phase consisting of the quark phase (stable) and the hadron phase (unstable). At the temperature $T=T_{\text {cont }}(\rho)$ the system experiences a continuous transition to the pure chirally symmetric phase.
\end{abstract}

PACS: 11.30.Rd, 12.38.Aw, 25.75.Nq

It is well known, that the chiral symmetry is valid in perturbative quantum chromodynamics (QCD) with massless quarks. It is expected also, that the chiral symmetry takes place in full-perturbative and nonperturbative QCD at high temperatures, $(T \gtrsim 200 \mathrm{MeV})$, if heavy quarks $(c, b, t)$ are ignored. The chiral symmetry is strongly violated, however, in hadronic matter, i.e. in QCD at $T=0$ and low density. What is the order of phase transition between two phases of QCD with broken and restored chiral symmetry at variation of temperature and density is not completely clear now. There are different opinions about this subject (for a detailed review see Ref. [1, 2] and references therein).

We would like to consider here the influence of baryon density on the chiral phase transition in hadronic matter. Kogan, Kovner and Tekin [3] have suggested the idea, that baryons may initiate the restoration of chiral symmetry, if their density is high - when roughly half of the volume is occupied by baryons. The physical argument in favour of this idea comes from the hypothesis (supported by calculation in chiral soliton model of nucleon [4 ), that inside the baryon the chiral condensate has the sign opposite to that in vacuum. This hypothesis is not proved. Even more, it is doubtful, that the concept of quark condensate inside the nucleon can be formulated in a correct way in quantum theory. But the idea on the strong influence of baryon density on the chiral phase transition looks very attractive. For this reason no assumption on the driving mechanism of chiral phase transition at zero baryon density will be done in this paper. The problem, under consideration is: how the phase transition changes in the presence of baryons. The content of the paper closely follows ref.5.

We discuss the phase transitions in QCD with two massless quarks, $u$ and $d$. Many lattice calculations [6. 7. 8, 9] indicate, that at zero chemical potential the phase transition is of the second order. It will be shown below, that the account of baryon density drastically changes the situation and the transition becomes of the first order, and, at high density, the matter is always in the chirally symmetric phase.

Let us first consider a case of the zero baryonic density and suppose that the phase transition from chirality violating phase to the chirality conserving one is of the second order. The second order phase transition is, generally, characterized by the order parameter $\eta$. The order parameter is a thermal average of some operator which may be chosen in various ways. The physical results are independent on the choice of the order parameter. In QCD the quark condensate, $\eta=|\langle 0|\bar{u} u| 0\rangle|=|\langle 0|\bar{d} d| 0\rangle| \geq 0$, may be taken as such parameter. In the confinement phase the quark condensate is non-zero while in the deconfinement phase it is vanishing.

The quark condensate has the desired properties: as it was demonstrated in the chiral effective theory [10] 11, $\eta$ decreases with the temperature increasing and an extrapolation of the curve $\eta(T)$ to the higher temperatures indicates, that $\eta$ vanishes at $T=T_{c}^{(0)} \approx 180 \mathrm{MeV}$. Here the superscript " 0 " indicates that 
the critical temperature is taken at zero baryon density. The same conclusion follows from the lattice calculations 6, 9, 12, where it was also found that the chiral condensate $\eta$ decreases with increase of the chemical potential 13, 14.

Apply the general theory of the second order phase transitions [15] and consider the thermodynamical potential $\Phi(\eta)$ at the temperature $T$ near $T_{c}^{(0)}$. Since $\eta$ is small in this domain, $\Phi(\eta)$ may be expanded in $\eta$ :

$$
\Phi(\eta)=\Phi_{0}+\frac{1}{2} A \eta^{2}+\frac{1}{4} B \eta^{4}, \quad B>0 .
$$

For a moment we neglect possible derivative terms in the potential.

The terms, proportional to $\eta$ and $\eta^{3}$ vanish for general reasons [15]. In QCD with massless quarks the absence of $\eta$ and $\eta^{3}$ terms can be proved for any perturbative Feynman diagrams. At small $t=T-T_{c}^{(0)}$ the function $A(t)$ is linear in $t: A(t)=a t, a>0$. If $t<0$ the thermodynamical potential $\Phi(\eta)$ is minimal at $\eta \neq 0$, while at $t>0$ the chiral condensate vanishes $\eta=0$. At small $t$ the $t$-dependence of the coefficient $B(t)$ is inessential and may be neglected. The minimum, $\bar{\eta}$, of the thermodynamical potential can be found from the condition, $\partial \Phi / \partial \eta=0$ :

$$
\bar{\eta}= \begin{cases}\sqrt{-a t / B}, & t<0 \\ 0, & t>0\end{cases}
$$

It corresponds to the second order phase transition since the potential is quartic in $\eta$ and - if the derivative terms are included in the expansion - the correlation length becomes infinite at $T=T_{c}^{(0)}$.

Turn now to the case of the finite, but small baryon density $\rho$ (by $\rho$ we mean here the sum of baryon and anti-baryon densities). For a moment, consider only one type of baryons, i.e. the nucleon. The temperature of the phase transition, $T_{\mathrm{ph}}$, is, in general, dependent on the baryon density, $T_{\mathrm{ph}}=T_{\mathrm{ph}}(\rho)$, with $T_{\mathrm{ph}}(\rho=0) \equiv T_{c}^{(0)}$ At $T<T_{\mathrm{ph}}(\rho)$ the term, proportional to $E \rho$, where $E=\sqrt{p^{2}+m^{2}}$ is the baryon energy, must be added to the thermodynamical potential (11). As was shown in [16, 17] the nucleon mass $m$ (as well as the masses of other baryons) arises due to the spontaneous violation of the chiral symmetry and is approximately proportional to the cubic root of the quark condensate: $m=c \eta^{1 / 3}$, with $c=\left(8 \pi^{2}\right)^{1 / 3}$ for a nucleon. At small temperatures $T$ the baryon contribution to $\Phi$ is strongly suppressed by the Boltzmann factor $e^{-E / T}$ and is negligible. Below we assume that the proportionality $m \sim \eta^{1 / 3}$ is valid in a broad temperature interval. Arguments in favor of such an assumption are based on the expectation that the baryon masses vanish at $T=T_{\mathrm{ph}}(\rho)$ and on the dimensional grounds. Near the phase transition point $E=\sqrt{p^{2}+m^{2}} \approx p+c^{2} \eta^{2 / 3} /(2 p)$. At $\eta \rightarrow 0$ all baryons are accumulating near zero mass and a summation over all baryons gives us - instead of eq. (1) - the following:

$$
\Phi(\eta, \rho)=\Phi_{0}+\frac{1}{2} a t \eta^{2}+\frac{1}{4} B \eta^{4}+C \eta^{2 / 3} \rho,
$$

where $C=\sum_{i} c_{i}^{2} /\left(2 p_{i}\right)$. The term $\rho \sum_{i} p_{i}$ is absorbed into $\Phi_{0}$ since it is independent on the chiral condensate $\eta$. The typical momenta are of the order of the temperature, $p_{i} \sim T$. Thus, Eq. (33) is valid in the region $\eta \ll T^{3}$. In the leading approximation the term $C$ can be considered as independent on the temperature at $T \sim T_{c}^{(0)}$.

Due to the last term in Eq. (3) the thermodynamical potential always have a local minimum at $\eta=0$ since the condensate $\eta$ is always non-negative. At small $t<0$ there also exists a local minimum at $\eta>0$, which is a solution of the equation:

$$
\frac{\partial \Phi}{\partial \eta} \equiv\left(a t+B \eta^{2}\right) \eta+\frac{2}{3} C \rho \eta^{-1 / 3}=0 .
$$

At small enough baryon density $\rho$, Eq. (4) [visualized in Figure 1(a)] has, in general, two roots, $\eta_{1}<\eta_{0}$ and $\eta_{2}>\eta_{0}$, where $\eta_{0}=(-a t / 3 B)^{1 / 2}$ is the minimum of the first term in the right-hand side of Eq. (4). The calculation of the second derivative $\partial^{2} \Phi / \partial \eta^{2}$ shows that the second root $\eta_{2}$ (if exists) corresponds to minimum of $\Phi(\eta)$ and, therefore, is a local minimum of $\Phi$. The point $\eta=\eta_{1}$ corresponds to a local maximum of the thermodynamical potential since at this point the second derivative is always non-positive.

The thermodynamical potential $\Phi(\eta, \rho)$ at (fixed) non-zero baryon density $\rho$ has the form plotted in Figure 1(b). At low enough temperatures (curve $T_{1}$ ) the potential has a global minimum at $\eta>0$ and system resides in the chirally broken (hadron) phase. As temperature increases the minima at $\eta=0$ and 


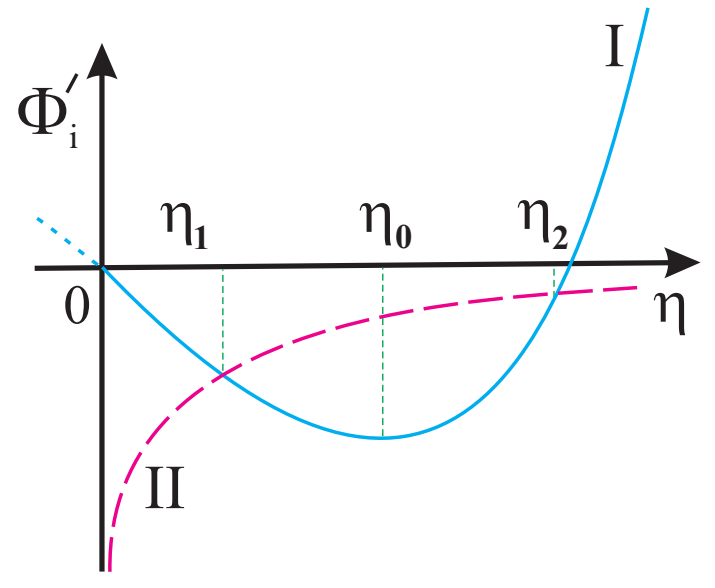

(a)

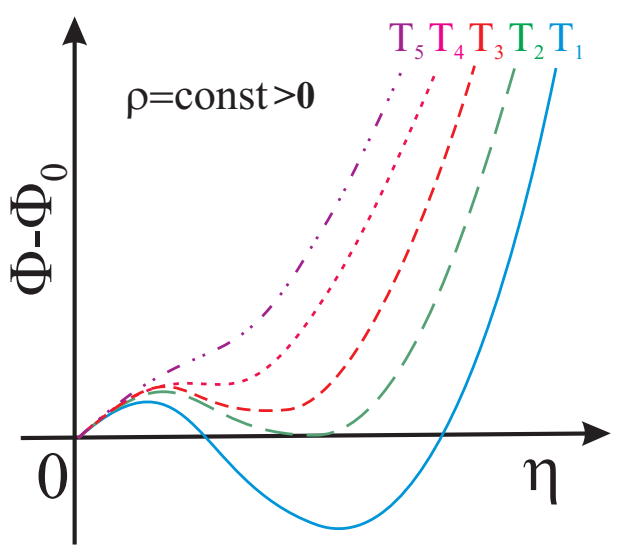

(b)

Figure 1: Figure 1: (a) Graphical representation of Eq. (4): "I" is the first term and "II" the second term (with the opposite sign) in the r.h.s. of the equation. (b) The thermodynamic potential (3) vs. the chiral condensate at a fixed baryon density $\rho>0$. At low enough temperatures, $T=T_{1}$, the system resides in the chirally broken (hadron) phase. The first order phase transition to the quark phase takes place at $T_{\mathrm{ph}}=T_{2}>T_{1}$. At somewhat higher temperatures, $T_{3}>T_{\mathrm{ph}}$ the system is in a mixed state. The temperature $T_{4} \equiv T_{\text {cont }}$ corresponds to a continuous transition to the pure quark phase, in which the thermodynamic potential has the form $T_{5}$.

at $\eta=\bar{\eta}_{2}>0$ becomes of equal height (curve $T_{2} \equiv T_{\mathrm{ph}}$ ). At this point the first order phase transition to the quark phase takes place. At somewhat higher temperatures, $T=T_{3}>T_{\mathrm{ph}}$, the $\eta>0$ minimum of the potential still exist but $\Phi(\eta=0)<\Phi\left(\bar{\eta}_{2}\right)$. This is a mixed phase, in which the bubbles of the hadron phase may still exist. However, as temperature increases further, the second minimum disappears (curve $\left.T_{4} \equiv T_{\text {cont }}\right)$. This temperature corresponds to a continuous transition to the pure quark phase, in which the thermodynamic potential has the form $T_{5}$.

Let us calculate the temperature of the phase transition, $T_{\mathrm{ph}}(\rho)$, at non-zero baryon density $\rho$. The transition corresponds to the curve $T_{2}$ in Figure $(\mathrm{b})$, which is defined by the equation $\Phi\left(\bar{\eta}_{2}, \rho\right)=\Phi(\eta=0, \rho)$, where $\bar{\eta}_{2}$ is the second root of Eq. (4) as discussed above. The solution is

$$
T_{\mathrm{ph}}(\rho)=T_{c}^{(0)}-\frac{5}{a}\left(\frac{2 C \rho}{3}\right)^{3 / 5}\left(\frac{B}{4}\right)^{2 / 5},
$$

and the second minimum of the thermodynamic potential is at $\bar{\eta}_{2}=\left[4 a\left(T^{(0)}-T_{\mathrm{ph}}(\rho)\right) /(5 B)\right]^{1 / 2}$.

At a temperature slightly higher than $T_{\mathrm{ph}}(\rho)$ the potential is minimal at $\eta=0$, but it has also an unstable minimum at some $\eta>0$. The existence of metastable state is also a common feature of the first order phase transition (e.g., the overheated liquid in case of liquid-gas system). With a further increase of the density $\rho$ (at a given temperature) the intersection of the two curves in Figure 1(a) disappears and the two curves only touch one another at one point $\eta=\bar{\eta}_{4}$. At this temperature a continuous transition (crossover) takes place. The corresponding potential has the characteristic form denoted as $T_{4}$ in Figure 1(a). The temperature $T_{4} \equiv T_{\text {cont }}$ is defined by the condition that the first (4) and the second derivatives of Eq. (3) vanish:

$$
T_{\text {cont }}(\rho)=T_{c}^{(0)}-\frac{5}{a}\left(\frac{2 C \rho}{9}\right)^{3 / 5}\left(\frac{B}{2}\right)^{2 / 5},
$$

and the value of the chiral condensate, where the second local minimum of the potential disappears is given by $\bar{\eta}_{4}=\left[2 a\left(T_{\text {cont }}(\rho)-T_{c}^{(0)}\right) /(5 B)\right]^{1 / 2}$. At temperatures $T>T_{\text {cont }}(\rho)$ the potential has only one minimum and the matter is in the state with the restored chiral symmetry. Thus, in QCD with massless quarks the type of phase transition with the restoration of the chiral symmetry strongly depends on the value of baryonic 
density $\rho$. At a fixed temperature, $T<T_{c}^{(0)}$, the phase transition happens at a certain critical density, $\rho_{\text {ph }}$. According to Eq. (5. the critical density has a kind of a "universal" dependence on the temperature, $\rho_{\mathrm{ph}}(T) \propto\left[T_{c}^{(0)}-T\right]^{5 / 3}$, the power of which does not depend on the parameters of the thermodynamic potential, $a$ and $B$.

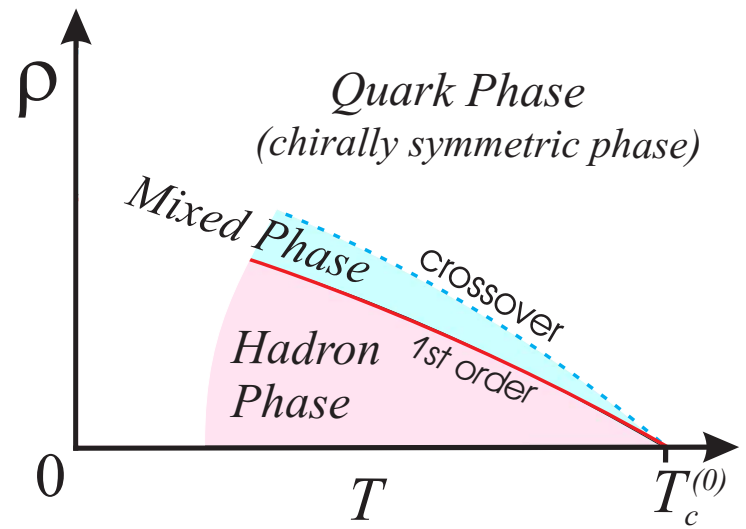

(a)

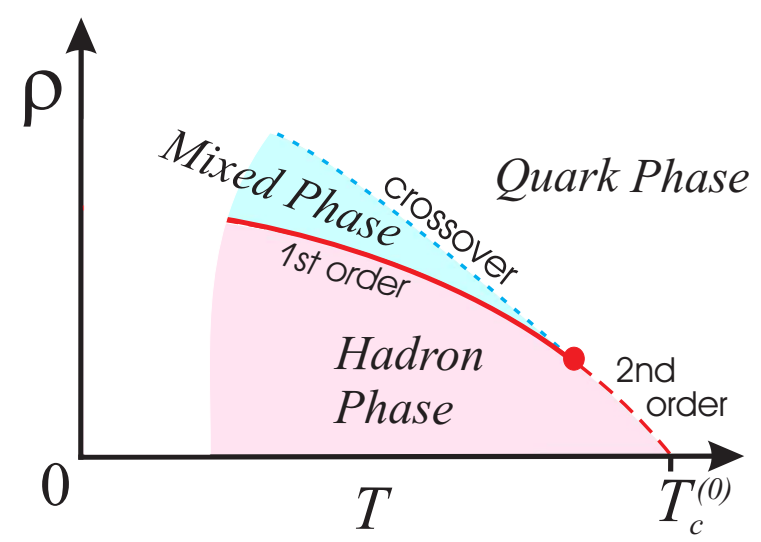

(b)

Figure 2: Figure 2: The qualitative phase diagram at finite baryon density and temperature based on the analysis (a) without and (b) with indication of the approximate 2-nd order transition domain.

The expected phase diagram is shown qualitatively in Figure 2(a). This diagram does not contain an end-point which was found in lattice simulations of the QCD with a finite chemical potential 18, 19. We expect that this happens because in our approach a possible influence of the confinement on the order of the chiral restoration transition was ignored. Intuitively, it seems that at low baryon densities such influence is absent indeed: the deconfinement phenomenon refers to the large quark-anti-quark separations while the restoration of the of the chiral symmetry appears due to fluctuations of the gluonic fields in the vicinity of the quark. However, the confinement phenomenon dictates the value of the baryon size which can not be ignored at high baryon densities, when the baryons are overlapping. If the melting of the baryons happens in the hadron phase depicted in Figure 2 (a), then at high enough density the nature of the transition could be changed. This may give rise in appearance of the end-point observed in Ref. 18, 19. The domain where the inequality $|a t| \gg C \rho \eta^{2 / 3}, \rho \neq 0$ is fulfilled, has specific features. In this domain the phase transition looks like a smeared second order phase transition: the specific heat has (approximately) a discontinuity at the phase transition point, $\Delta C_{p}=a^{2} T_{c} / B$. The correlation length increases as $\left(T-T_{c}^{(0)}\right)^{-1 / 2}$ at $T-T_{c}^{(0)} \rightarrow 0$. The latter arises if we include the derivative terms in the effective thermodynamical potential. The phase diagram with this domain indicated may look as it is shown in Figure 2(b). Note that the applicability of our considerations is limited to the region $\left|T-T_{c}^{(0)}\right| / T_{c}^{(0)} \ll 1$ and low baryon densities.

In the real QCD the massive heavy quarks (the quarks $c, b, t$ ) do not influence on this conclusion, since their concentration in the vicinity of $T \approx T_{c}^{(0)} \sim 200 \mathrm{MeV}$ is small. However, the strange quarks, the mass of which $m_{s} \approx 150 \mathrm{MeV}$ is just of order of expected $T_{c}^{(0)}$, may change the situation. This problem deserves further investigation.

This work was supported in part by INTAS grant 2000-587, RFBR grants 03-02-16209, 01-02-17456, 03-02-04016 and MK-4019.2004.2.

\section{References}

[1] A.V.Smilga, "Lectures On Quantum Chromodynamics", in Handbook of QCD, Boris Ioffe Festschrift, ed.by M.Shifman, World Scientific, Singapore 2001, vol.3, p.2033.

[2] K. Rajagopal and F. Wilczek, "The Condensed Matter Physics of QCD", ibid., p. 2061.

[3] I.I.Kogan, A.Kovner and B.Tekin, Phys.Rev. D63, 116007 (2001). 
[4] D.Diakonov and V.Petrov, Handbook of QCD, Festschrift in honor of B.L.Ioffe, ed by M.Shifman, World Scientific, 2001, v.1, p.359.

[5] M.N.Chernodub and B.L.Ioffe, Pisma v ZhETF, 79, 742 (2004), hep-ph/0405042

[6] F. Karsch, Phys. Rev. D 49, 3791 (1994).

[7] F. Karsch and E. Laermann, Phys. Rev. D 50, 6954 (1994).

[8] S. Aoki et al. [JLQCD Collaboration], Phys. Rev. D 57, 3910 (1998).

[9] A. Ali Khan et al. [CP-PACS Collaboration], Phys. Rev. D 63, 034502 (2001).

[10] H.Leutwyler, Nucl. Phys. B (Proc.Suppl.) 4, 248 (1988).

[11] P.Gerber and H.Leutwyler, Nucl.Phys. B 321, 387 (1989).

[12] F. Karsch, Nucl. Phys. A 590, 367C (1995).

[13] J. B. Kogut, D. K. Sinclair, S. J. Hands and S. E. Morrison, Phys. Rev. D 64, 094505 (2001).

[14] J. B. Kogut, D. Toublan and D. K. Sinclair, Nucl. Phys. B 642, 181 (2002).

[15] L.Landau and E.Lifshitz, Statistical Physics, part 1, Pergamon Press, Oxford, 3-d edition, 1977.

[16] B.L.Ioffe, Nucl.Phys. B188, 317 (1981), E191, 591 (1981).

[17] V.M.Belyaev and B.L.Ioffe, ZhETF 83876 (1982), 84, 1236 (1983).

[18] Z. Fodor and S. D. Katz, JHEP 0203, 014 (2002).

[19] Z. Fodor and S. D. Katz, hep-lat/0402006 